\title{
Electrically Addressable Hybrid Architectures of Zinc Oxide Nanowires Grown on Aligned Carbon Nanotubes
}

\author{
By Jong G. Ok, Sameh H. Tawfick, K. Anne Juggernauth, Kai Sun, \\ Yongyi Zhang, and A. John Hart*
}

The fabrication and characterization of hybrid architectures of $\mathrm{ZnO}$ nanowires (ZNWs) grown on organized carbon nanotubes (CNTs), by a two-step chemical vapor deposition (CVD) process involving CNT growth from a hydrocarbon source followed by ZNW growth using a Zn metal source, is reported. The ZNWs grow uniformly and radially from individual CNTs and CNT bundles, and the aligned morphology of the CNTs is not disturbed by the ZNW growth process. The nucleation and growth of ZnO crystals on CNTs are analyzed in relation to the classical vapor-solid mechanism. Importantly, the CNTs make uniform and distributed electrical contact to the ZNWs, with up to a 1000-fold yield advantage over conventional ZNW growth on a flat substrate. Hybrid ZNW/CNT sheets are fabricated by scalable CVD, rolling, and printing methods; and their electrical properties, which are governed by transport through the anisotropic CNT network, are characterized. Functional interaction between the ZNWs and CNTs is demonstrated by photoconductive behavior and photocurrent generation of the hybrid material under UV illumination. There is significant future opportunity to extend these processing methods to fabricate other functional oxides on CNTs, and to build devices that harness the attractive properties of ZNWs and CNTs with high volumetric efficiency over large areas.

\section{Introduction}

Hierarchically structured materials having a high density of nanoscale junctions between complimentary materials (e.g., conductors and semiconductors) along with large interfacial areas

[*] Prof. A. J. Hart, J. G. Ok, S. H. Tawfick, K. A. Juggernauth, Y. Zhang Mechanosynthesis Group

Department of Mechanical Engineering, University of Michigan

2350 Hayward Street, Ann Arbor, MI 48109 (USA)

E-mail: ajohnh@umich.edu

K. A. Juggernauth

Macromolecular Science and Engineering Research Center

Department of Materials Science and Engineering, University of Michigan

2455 Hayward Street, Ann Arbor, MI 48109 (USA)

Dr. K. Sun

Electron Microbeam Analysis Laboratory

Department of Materials Science and Engineering, University of Michigan

2455 Hayward Street, Ann Arbor, MI 48109 (USA) will be essential components of energy conversion and storage technologies that will bolster the future of our society. To this end, rationally designing, manufacturing, and studying the performance of selfsimilar assemblies of nanostructures at multiple length scales is indispensable towards understanding the capabilities and limitations of novel materials. Applications range from nanoscale chemical and biological sensors to electrodes for next generation batteries, capacitors, and photovoltaics. Scalable manufacturing of energy conversion and storage materials demands both engineering of individual nanostructures, and assembly of large numbers of complementary nanostructures into truly three-dimensional (3D) architectures that meet application-specific needs. ${ }^{[1]}$

Recent efforts have explored the synthesis and applications of myriad one-dimensional (1D) nanostructures made of individual materials, ${ }^{[2]}$ as well as nanoscale heterostructures that combine the attributes of multiple materials. For example, "superlattice" nanowires (NWs) with alternating material segments are made by cycling the precursor chemistry during growth $^{[3]}$, or by sequential filling of porous templates. ${ }^{[4,5]}$ Coreshell architectures are made by sequentially depositing nanoscale films on the outer surfaces of NWs, and these can function individually as photovoltaic cells. ${ }^{[6]}$ Branched structures are made by seeding the outer surfaces of NWs with catalyst to cause additional NWs or nanotubes (NTs) to grow in a simultaneous or sequential fashion. ${ }^{[7,8]}$ Also, many types of metal, oxide, polymer, and semiconductor nanoparticles (NPs) have been placed on the outer surface of carbon nanotubes (CNTs). ${ }^{[9-12]}$ However, while extensive research has demonstrated the unique size and structure-dependent properties of these and many other nanostructured "building blocks", relatively few scalable approaches exist for creating functional hybrid architectures in three dimensions.

A typical and versatile construct of $1 \mathrm{D}$ nanostructures is a vertically aligned array, where a large number (typically greater than $10^{10} \mathrm{~cm}^{-2}$ ) of NTs or NWs is grown perpendicular to the surface of a flat substrate. Depending on the characteristics of the structures and synthesis methods, the vertical alignment is 
achieved by crystallographic interactions with the substrate and/or by crowding among the structures during the initial stages of growth. This construct is attractive due to the high surface area of the nanostructures yet is limited by their length and by sometimes conflicting requirements for the structures both to convert or store energy carriers (e.g., by charge separation or intercalation), and to transport electrons to the substrate electrode. Therefore, a promising hybrid 3D construct could be created using an array of vertically aligned and electrically conducting nanostructures as the substrate for a second type of nanostructure having complementary properties.

$\mathrm{CNTs}^{[13]}$ are particularly attractive as scaffolds and templates for forming, connecting, and addressing other types of nanostructures since they are electrically conductive (all multiwalled CNTs (MWNTs) and statistically 33\% of single-walled CNTs) and mechanically robust, and their surfaces can be modified chemically. ${ }^{[12]}$ Further, vertically aligned CNT "forests" can be grown on diverse substrates including silicon wafers, ${ }^{[14]}$ metal foils, ${ }^{[15]}$ and advanced fibers, ${ }^{[16]}$ and chemical vapor deposition (CVD) methods in combination with ordinary lithography can create patterned and molded ${ }^{[17]}$ CNT forests over length scales ranging from micrometers to millimeters. Consequently, there has been wide study of CNT forests as electrodes for capacitors, ${ }^{[18]}$ lithium ion batteries, ${ }^{[10]}$ photovoltaic cells, ${ }^{[19]}$ and flexible energy storage devices. ${ }^{[1]}$

Zinc oxide $(\mathrm{ZnO}) \mathrm{NWS}^{[20]}$ have been investigated for applications including light-emitting devices ${ }^{[21]}$ and vibrational energy harvesters. ${ }^{[22]} \mathrm{ZnO}$ NWs (herein called ZNWs) have a wide bandgap $(\approx 3.37 \mathrm{eV})$, large exciton binding energy $(\approx 60 \mathrm{meV})$, and a non-central symmetric wurtzite crystal structure. They also exhibit UV photoluminescence (PL) ${ }^{[21]}$ and piezoelectricity. ${ }^{[22]}$ However, despite recent progress in the synthesis of ZNW arrays by high temperature vapor-liquid-solid (VLS) ${ }^{[20]}$ and vapor-solid (VS), ${ }^{[23]}$ as well as by low temperature aqueous solution methods, ${ }^{[24]}$ the performance and practical feasibility of resulting $\mathrm{ZnO}$-based devices is limited by the lengths of ZNWs which are typically no more than 1-20 $\mu \mathrm{m}$. As a result, the power output of ZnO-based energy harvesters has been scaled up by stacking and wiring multiple substrates each holding NW arrays. ${ }^{[25]}$ While this is effective, the relatively short NW length means that the volumetric performance of such a device is dominated by the substrate size, and device fabrication and assembly requires multiple delicate sequences.

In this paper, we introduce a versatile and scalable method for building hybrid 3D architectures of oxide NWs on vertically aligned CNTs. The hybrid structures are made by a two-step CVD process involving growth of vertically aligned CNTs from catalyst NPs on a silicon wafer, followed by catalyst-free NW growth on the CNT surfaces by thermal evaporation of a pure metal source in the presence of oxygen. This process is demonstrated by growing ZNWs on vertically aligned MWNTs, as shown in Figure 1 . The ZNWs uniformly and conformally coat the surfaces of individual CNTs and CNT bundles throughout CNT forests. Importantly, the CNTs withstand the oxidative growth process for ZNWs and establish electrical contact to the ZNWs. Other approaches to fabricating complementary nanostructures with CNTs include

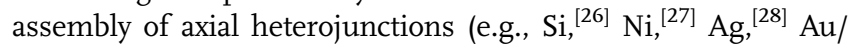
$\mathrm{Cu},{ }^{[5]}$ or $\mathrm{Sn}^{[29]}$ ) and synthesis of nanostructures (e.g., $\mathrm{MnO}_{2},{ }^{[30]}$ $\mathrm{GaN},{ }^{[31]} \mathrm{Pt},{ }^{[32]}$ and $\mathrm{TiO}_{2}{ }^{[33]}$ ) on the surfaces of CNTs. Fabrication of
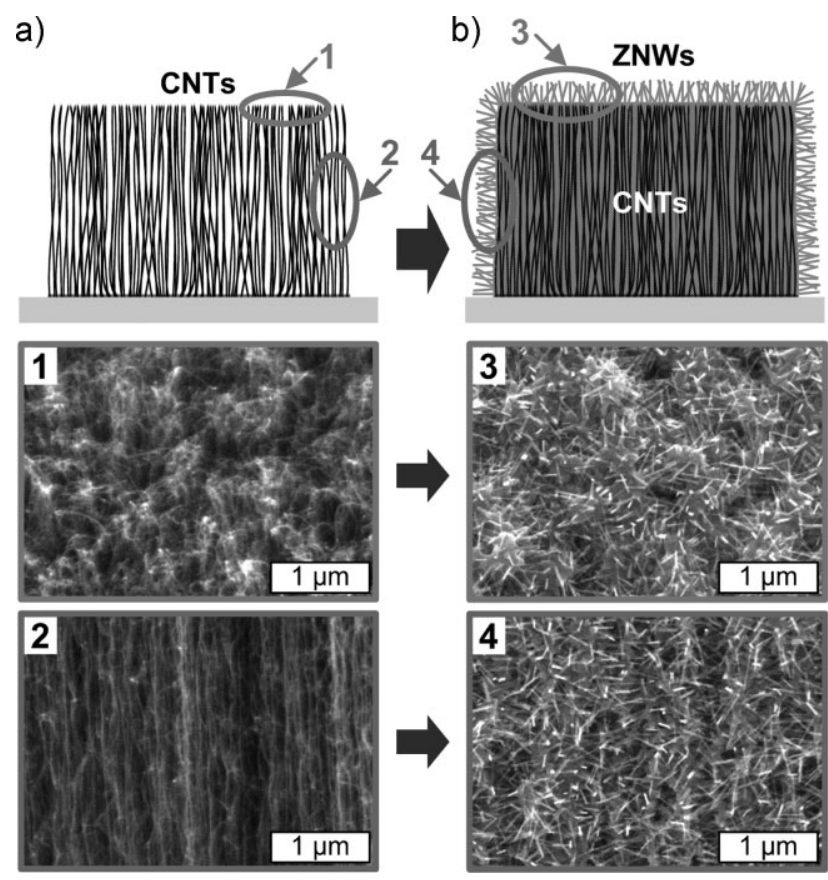

Figure 1. Growth of ZNWs on vertically aligned CNT forests. Schematic illustrations and SEM images show CNT forests before (a) and after (b) ZNW growth. SEM images were all taken from $45^{\circ}$ tilted view, as indicated by arrows. ZNW growth is shown on both tangled CNT morphology of the top surface of the forest $(1,3)$ and the aligned CNT morphology of the forest sidewall $(2,4)$.

CNT-based axial heterostructures often requires the step of template preparation (e.g., anodized aluminum oxide ${ }^{[5,27,28]}$ ) and later removal of the template. Additionally most methods to attach or grow other nanostructures onto CNTs have used solution chemistry which is advantageous due to its low temperature but can undesirably alter the morphology of the forest due to capillary forces.

We demonstrate scalable processing of vertically aligned hybrid ZNW/CNT forests, and laterally aligned ZNW/CNT thin films that can be integrated with lithographic processing. These novel materials have anisotropic electrical conductivity due to the hierarchical organization of the ZNWs on the CNT forest, and rapid photoelectric response due to the intimate contact between the nanostructured $\mathrm{ZnO}$ and the conductive CNTs.

\section{Synthesis and Characterization of Hybrid ZNW/CNT Forests}

\subsection{Growth of ZnO NWs on CNTs}

In order to create hybrid NT/NW architectures by simultaneously or sequentially performing two CVD processes, the respective process conditions and structures must be chemically and thermally compatible. Incidentally, we first learned that a typical VLS ZNW growth process ${ }^{[34]}$ using a $\mathrm{ZnO}$ powder source and $\mathrm{Au}$ catalyst is not suitable for creating hybrid ZNW/CNT structures, as the CNTs are destroyed (Fig. 1Sa in the Supporting Information) 

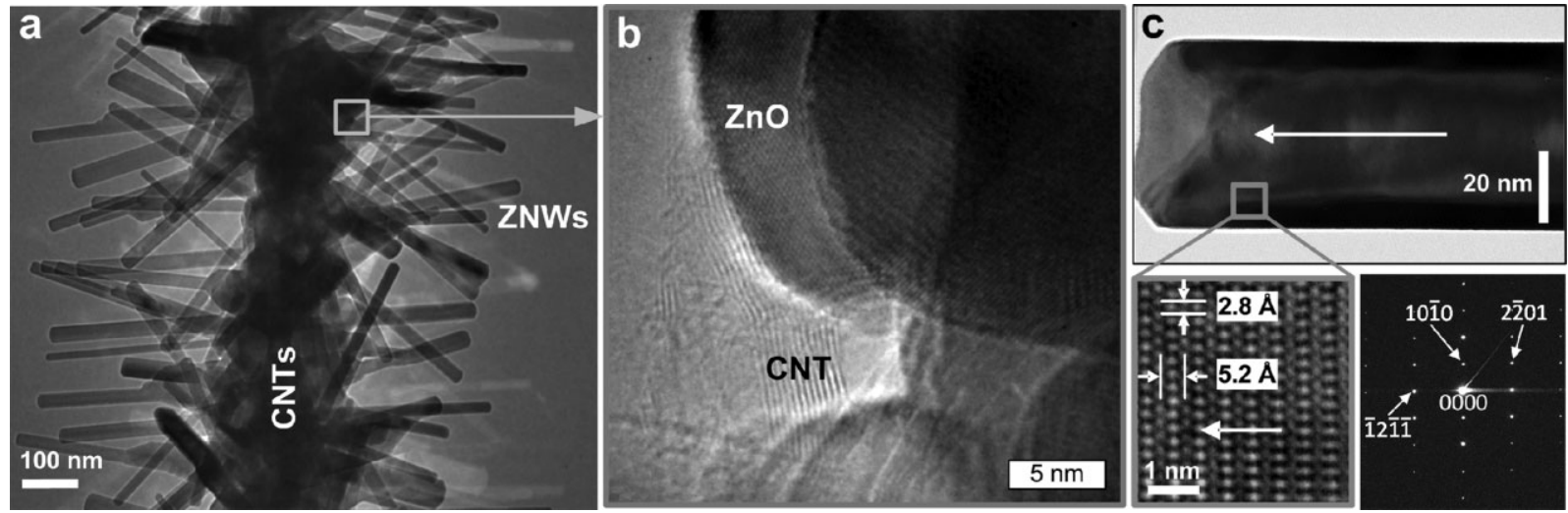

Figure 2. TEM images of a hybrid ZNW/CNT bundle: a) plain view of ZNW/CNT bundle with core diameter $\approx 100 \mathrm{~nm}$ and ZNW diameters $\approx 10-50 \mathrm{~nm}$; b) close view of the $\mathrm{ZnO}-\mathrm{CNT}$ interface showing intimate relationship between graphitic MWNT walls and ZnO nanocrystals; c) view of the tip of an individual ZNW. The lattice-resolved image and accompanying electron diffraction pattern of the ZNW in (c) verify the typical wurtzite crystal structure and c-axis [0001] growth direction.

due to the required combination of high temperature $\left(950^{\circ} \mathrm{C}\right)$ in an oxidizing environment. Further, an attempt to grow CNTs on ZNWs grown by the $\mathrm{ZnO}$ powder method failed because $\mathrm{ZNW}$ s are etched by hydrogen under the typical CNT growth conditions (Fig. $\mathrm{S} 1 \mathrm{~b})$. Hydrothermal growth of ZNWs can be conducted at very low temperatures $\left(70-200{ }^{\circ} \mathrm{C}\right)$ in liquid solution, and ZNWs were previously grown on CNTs by a hydrothermal method:; ${ }^{[5]}$ however, this process requires deposition of a nanocrystalline $\mathrm{ZnO}$ seed layer on the CNTs prior to ZNW growth, and needs a very long growth time (i.e., 12-48 h). More importantly, surface tension forces from the $\mathrm{ZnO}$ growth solution inevitably cause elastocapillary aggregation of the $\mathrm{CNTs}^{\left[{ }^{[36]}\right.}$ and this can undesirably alter the network morphology and prevent achievement of a wellorganized hybrid array.

Accordingly, to facilitate synthesis of ZNWs without damage to the CNT substrate, we used a Zn foil source which has a much lower melting temperature $\left(\approx 420^{\circ} \mathrm{C}\right)$ and therefore creates sufficient $\mathrm{Zn}$ vapor ambient far below the oxidation temperature of CNTs. Using this approach, ZNW growth is performed in a conventional tube furnace (Fig. S2a) with a controlled low pressure atmosphere of mixed He and purified air. Air provides oxygen to form $\mathrm{ZnO}$, while $\mathrm{He}$ enables independent regulation of the $\mathrm{Zn}$ vapor concentration and downstream transport for control of NW growth. A similar technique previously achieved the growth of a ZNW arrays on planar oxide substrates ${ }^{[37]}$ without requiring a catalyst or seed layer. Unless otherwise noted, the structures shown in this paper were grown on MWNT forests for $20 \mathrm{~min}$ at $600^{\circ} \mathrm{C}$ and 6 Torr, with a flow of $17.5 \mathrm{sccm}$ air mixed with $20 \mathrm{sccm} \mathrm{He}$ Further information, including details of the CNT growth method, is given in the Experimental section.

CNT forests which are initially black in color appear light gray to the naked eye after ZNW growth. The ZNWs grow uniformly and radially from the CNTs within the forest, as shown in scanning electron microscope (SEM) images (Fig. 1). Notably, the aligned morphology of the CNTs is not disturbed by the ZNW growth process. Energy-dispersive X-ray spectroscopy (EDX) analysis (Fig. S3a) also confirms that the structures grown on CNTs consist of Zn and O. Further, grazing incidence X-ray diffraction (GI-XRD, Fig. $\mathrm{S} 3 \mathrm{~b})$ confirms that the coating is crystalline $\mathrm{ZnO}$.
ZNW growth penetrates uniformly into the CNT forest to a distance of approximately $15 \mu \mathrm{m}$; thus, growing ZNWs on CNT microstructures that are first grown from lithographically patterned catalysts can achieve fully 3D ZNW growth over areas. An example of this is shown in Figure S4, and demonstrates in principle that a wide variety of hierarchical designs can be built by our process.

\subsection{Structural Characterization}

Transmission electron microscopy (TEM) imaging of a hybrid ZNW/CNT bundle, shown in Figure 2, reveals precisely how the ZNWs extend from a nanocrystalline base layer that coats the CNTs. The ZNWs are approximately $300-500 \mathrm{~nm}$ long and 25$50 \mathrm{~nm}$ diameter, and notably this diameter is smaller than that of ZNWs typically grown on flat substrates from a powder source. ${ }^{[38]}$ As clearly observed in Figure $2 \mathrm{~b}$, the $\mathrm{ZnO}$ nanocrystals directly contact the outer walls of the CNTs. Lattice-resolved imaging and selected-area electron diffraction (SAED) of an individual ZNW (Fig. 2c) determines the lattice parameters a $\times \cos 30^{\circ}=2.8 \AA$ and $\mathrm{c}=5.2 \AA$, which are consistent with the exact values of $\mathrm{a}=3.25 \AA$ and $c=5.21 \AA .{ }^{[39]}$ Therefore, the ZNWs have the expected hexagonal wurtzite $\mathrm{ZnO}$ structure, and grow along the c-axis in the [0001] direction which is energetically favored. ${ }^{[40]}$

Additional imaging confirms the polydispersity of $\mathrm{ZnO}$ crystal orientation with respect to the CNTs. For example, Figure 3 shows that $\mathrm{ZnO}$ crystals can nucleate directly on the CNT surface, and can also homogeneously branch at equal angles. In both cases, $\mathrm{ZnO}$ grows along the c-axis. Scanning TEM (STEM) with electron energy loss spectroscopy (EELS) (Fig. 3d) discloses a sharp interface profile between $\mathrm{ZnO}$ (represented by the oxygen edge) and $\mathrm{C}$, where the transition between $\mathrm{O}$ and $\mathrm{C}$ occurs completely within a distance of $\approx 2.5 \mathrm{~nm}$. Establishing this intimate contact is necessary for transport across the ZNW/CNT interface, and thus is vital for devices and materials which combine the characteristic properties of multiple nanostructures.

To assess how the outer surfaces of CNTs behave as a substrate for ZNW growth, ZNWs were also grown on isolated groups of 


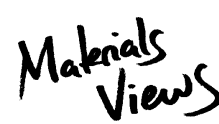

www.MaterialsViews.com
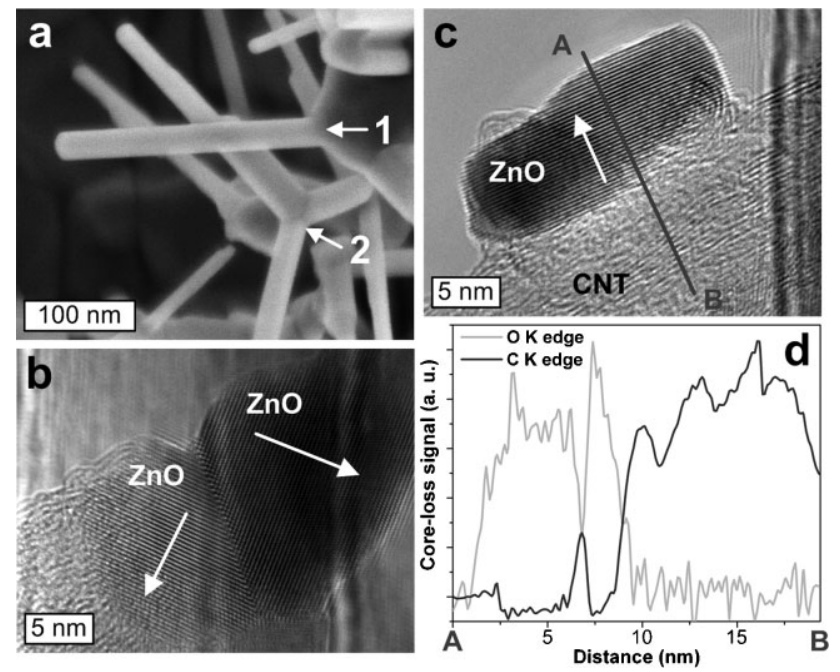

Figure 3. Growth of ZNWs from the interface of $\mathrm{ZnO}$ and a CNT. a) The high resolution (HR)-SEM image shows how ZNWs protrude (1) from a basal polycrystalline $\mathrm{ZnO}$ film that grows on the CNTs and how particular ZNWs meet at tetrahedral junctions emerging from the ZNW-coated CNT surface (2). TEM images demonstrate tetrahedral (b) and normal (c) growth of ZnO clusters on a CNT surface, which are to develop to ZNWs along the $\mathrm{c}$-axis apparently distinguishable for each cluster by the lattice structure, as indicated by arrows. EELS line scan profiles (d) of C (using its $\mathrm{K}$ edge at $284 \mathrm{eV}$ ) and $\mathrm{O}$ (using its $\mathrm{K}$ edge at $532 \mathrm{eV}$ ) were taken along the $\mathrm{A}-\mathrm{B}$ line across the $\mathrm{ZnO}-\mathrm{CNT}$ interface marked in (c), indicating a clear and chemically stable boundary.

CNTs, which were created by contact transfer of CNTs from a forest onto a copper grid prior to ZNW growth. SEM examination of these structures (Fig. 4) reveals that the CNTsurfaces are typically coated by a polycrystalline $\mathrm{ZnO}$ film, and that ZNWs extend from this base layer. We find that individual CNTs (or very small groups) act as suspended "strings" that support ZNW growth, and larger groupings of CNTs are coated with radially oriented ZNWs on their outer surfaces. Because a CNT forest contains isolated and bundled CNTs of varied aggregation, ${ }^{[41]}$ we expect similar ZNW/ CNT structures are present throughout the CNT forest. Thus, controlling the diameter, density, and hierarchical organization of CNTs within a forest using known methods ${ }^{[42]}$ of controlling the CNT growth catalyst and CVD conditions can determine the relative density of ZNWs in the final hybrid material. So far, we have found that the ZNW diameter does not noticeably change with the size of the CNT bundle.

\subsection{Photoluminescence Analysis}

PL spectroscopy provides additional insight into the crystalline quality of the ZNWs and the interactions between the ZNWs and CNTs. Figure 5 shows the room-temperature PL spectra of our hybrid ZNW/CNTmaterial, measured at $325 \mathrm{~nm}$ excitation. The PL spectra demonstrate a sharp and intense near-band edge UV emission peak at $397 \mathrm{~nm}$, and a broad deep-level green emission peak at $\approx 550 \mathrm{~nm}$. An as-grown, bare CNT sample does not show any PL sensitivity within the scanned wavelength range, which
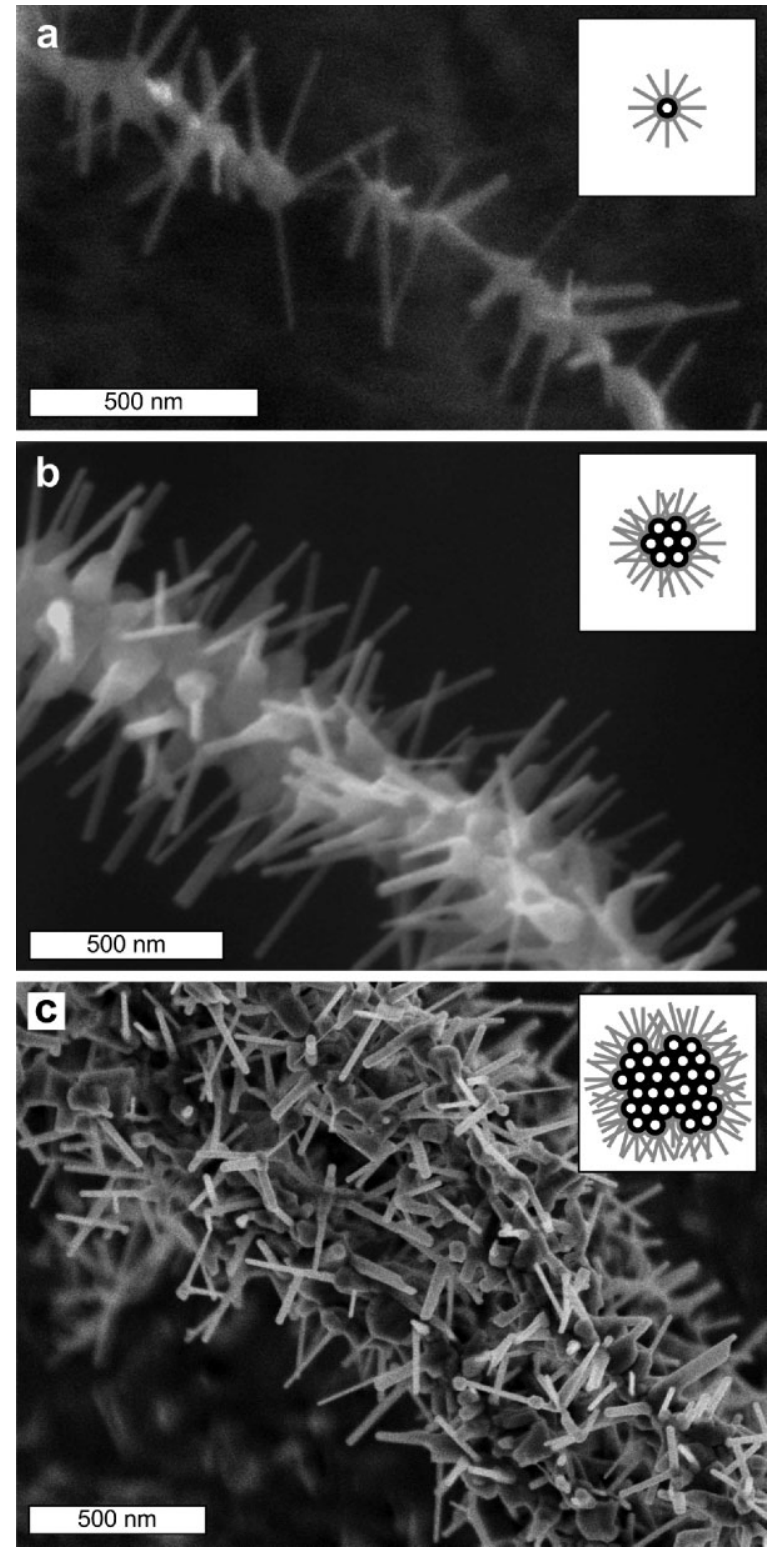

Figure 4. Conformal morphology of ZNW growth on the outer surfaces of aligned CNT assemblies: a) a small group of CNTs, having initial diameter $\approx 20 \mathrm{~nm}$; b) a larger group, having initial diameter $\approx 100 \mathrm{~nm}$ and ZNW length $\approx 500 \mathrm{~nm}$; and c) a successively larger group having an irregular outer surface, showing how ZNW growth locally follows the morphology of the CNT substrate.

confirms the peaks shown in the ZNW/CNT sample are from the $\mathrm{ZnO}$ nanostructures. Also, the PL spectra of ZNW/CNT structures are invariant when the excitation wavelength is varied. The series of noisy peaks observed at around $450-490 \mathrm{~nm}$ is an artifact of the adhesive backing which was used to mount the ZNW/CNT sample to the measurement substrate, as verified by the spectrum for the tape in Figure 5. The relatively sharp UV emission peak indicates that the sample is highly crystalline and of good structural quality. The broad decay observed here is also attributed to spectral overlap between the $\mathrm{ZnO}$ spectrum and artifacts from the mounting tape. 


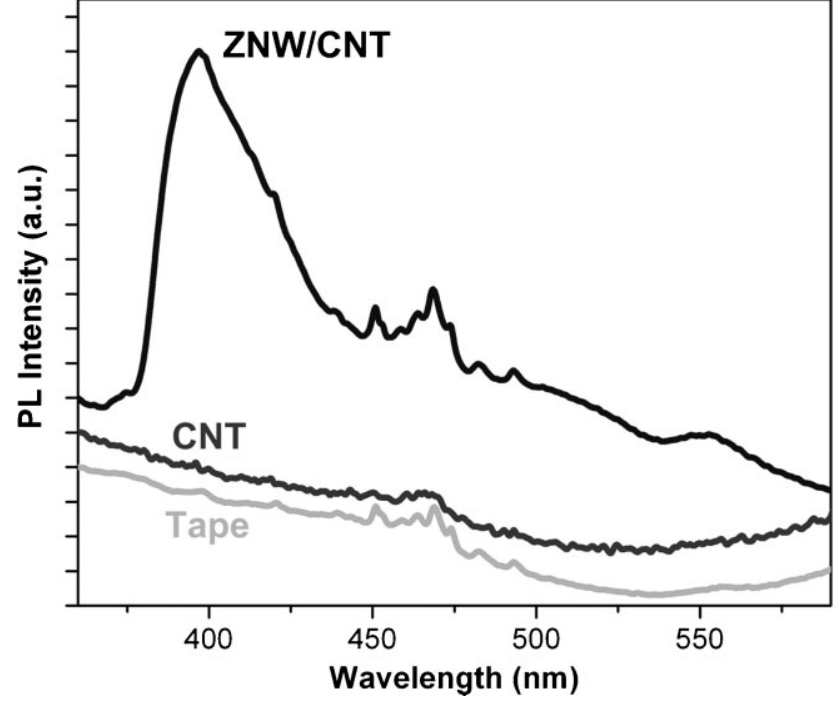

Figure 5. PL spectra of the top surfaces of a ZNW/CNT sample and a bare CNT sample having the same geometry $(1 \mathrm{~cm} \times 1 \mathrm{~cm}$ lateral dimensions $)$ taken at excitation wavelength of $325 \mathrm{~nm}$.

The deep-level visible wavelength $(550 \mathrm{~nm})$ peak is typically associated with defects in the $\mathrm{ZnO}$ structure ${ }^{[43]}$ such as oxygen vacancies ${ }^{[44]}$ and the broad peak centered at $525 \mathrm{~nm}$ is attributed to the recombination of photogenerated holes with ionized oxygen vacancies. ${ }^{[44]}$ It was also previously reported that the peak position of the green emission varies from $520 \mathrm{~nm}$ to $506 \mathrm{~nm}$ as the diameter of ZNWs varies from $100 \mathrm{~nm}$ to $25 \mathrm{~nm} .{ }^{[20]}$ This change was attributed to a greater fraction of oxygen vacancies present in the thinner nanowires, because the greater relative surface area of thinner nanowires favored a higher level of surface and subsurface oxygen vacancies. The broad peak for our sample is thus indicative of the polydispersity in ZNW diameters grown on CNTs. The PL characteristics of both the ZNWs and the underlying film, and the characteristics of the ZNW-CNT interface, must be investigated further to fully understand the PL behavior of the hybrid material.

Further, while not an absolute measure, the intensity ratio of the near-band edge adsorption peak in the UV range to the deep-level emission peak in the visible range provides an indication of the crystallinity of the system. ${ }^{[43,45]}$ Thus, a strong, intense UV peak and a weak, broad visible peak indicate that the hybrid ZNW/CNT structures are highly crystalline with low defects.

\section{Mechanism of ZnO Crystal Growth on CNTs}

It is evident that CNTs facilitate abundant formation of $\mathrm{ZnO}$ nanocrystals on their surfaces, which then form ZNWs by continued growth. According to the VS mechanism that has typically been studied for NW growth on flat substrates, ${ }^{[46]} \mathrm{Zn}$ atoms adsorb onto the substrate and migrate to defect sites (e.g., pits, protrusions) where they accumulate to form crystalline clusters. ${ }^{[47]}$ In our case, it is likely that $\mathrm{ZnO}$ nanocrystals form both on the surfaces of individual CNTs and in the nanoscale "grooves" between adjacent CNTs that are held closely by van der Waals interactions.

$\mathrm{Zn}$ is oxidized to $\mathrm{ZnO}$ upon interaction with the substrate, and as more $\mathrm{Zn}$ and $\mathrm{O}$ atoms are stacked on each $\mathrm{ZnO}$ grain, $\mathrm{ZNWs}$ grow preferentially along the [0001] direction which minimizes the total surface energy. After the stable clusters form, NW growth proceeds in the energetically favored [0001] direction. Previous research suggests NW growth is primarily fed by adsorption of adatoms onto the sidewalls of each NW and subsequent diffusion to the tips, where crystallization possibly occurs at advancing step edges arising from screw dislocations. ${ }^{[8,48]}$ If diffusion on the NW surface is much faster than self-nucleation of clusters on the NW surface, the NW lengthens much faster than its diameter increases, and this is a favorable condition for growth of long and slender NWs. ${ }^{[49]}$

Consistent with this mechanism, at short growth times (e.g., $5 \mathrm{~min}$ ), we observe short ZNWs along with isolated $\mathrm{ZnO}$ nanocrystals on the CNTs (Fig. S5). At longer times (20 min as shown in Figures 1 and 4), the ZNWs are longer and the CNT surfaces are fully covered by a polycrystalline $\mathrm{ZnO}$ film. We expect that ZNWs that grow at shallow angles with respect to the CNT surface are eventually incorporated in the $\mathrm{ZnO}$ film that coats the CNTs, and ZNWs that grow at larger angles to the substrate remain freestanding. As shown in Figure 3a, branching ${ }^{[50]}$ of the $\mathrm{ZnO}$ also occurs.

Thus, the mechanism of ZNW growth on CNTs is analogous to that on flat substrates, and the initial formation of $\mathrm{ZnO}$ nanocrystals can be further understood by a classical model of nucleation and growth. ${ }^{[51]} \mathrm{Zn}$ atoms adsorb and diffuse on the CNTs, forming ZnO nanocrystals that must exceed a critical size that dictates a decrease in their total free energy with continued growth. In other words, for a cluster of given size to be stable, its growth must create a net decrease in total free energy. This critical size is estimated by calculating the first derivative of the total free energy $(\Delta \mathrm{G})$ of a nanocrystal with respect to its radius $R$, as fully described in the Supporting Information. Modeling the cluster as a hemisphere on a flat substrate and considering the change in surface energy due to nanoscale curvature of the cluster (Fig. 6a), we find that the free energy of formation $(\Delta G)$ is

$\Delta G=\pi\left(3.3 \sigma_{1}+\sigma_{12}\right) R^{2}-\frac{2 \pi k T}{3 v} \ln \left(\frac{P}{P_{\infty}}\right) R^{3}$

Here, $\sigma_{1}$ is the cluster surface energy $(\mathrm{ZnO}), \sigma_{12}$ is the clustersubstrate interface energy ( $\mathrm{ZnO}-\mathrm{C}), v$ is the atomic volume, $k$ is Boltzmann's constant, $T$ is the absolute temperature of the substrate, $P$ is the pressure of the vapor precursor (determined from the source temperature), and $P_{\infty}$ is the equilibrium vapor pressure above the bulk surface at $T$. Also, by taking the first derivative of Equation (1), we obtain that the minimum stable radius $R^{*}$ (the critical radius) is

$R^{*}=\left(3.3 \sigma_{1}+\sigma_{12}\right) v / k T \ln \left(P / P_{\infty}\right)$

A critical limitation and simplification of this model is that the cluster contains only a single species; however, in our case Zn must interact with $\mathrm{O}$ to form $\mathrm{ZnO}$, and it is not precisely known when 
and where $\mathrm{Zn}$ is oxidized during the atomic processes of adsorption, diffusion, and cluster formation. Further, all atoms needed to form a crystal of stable size do not arrive simultaneously. Thus, the dynamic stability of a crystal is affected by the diffusion and residence times of the adatoms on the substrate, which are not known. This model also does not consider the effects of substrate

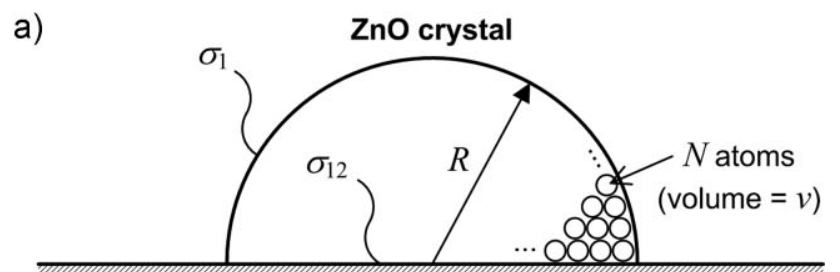

sumsinate

b)
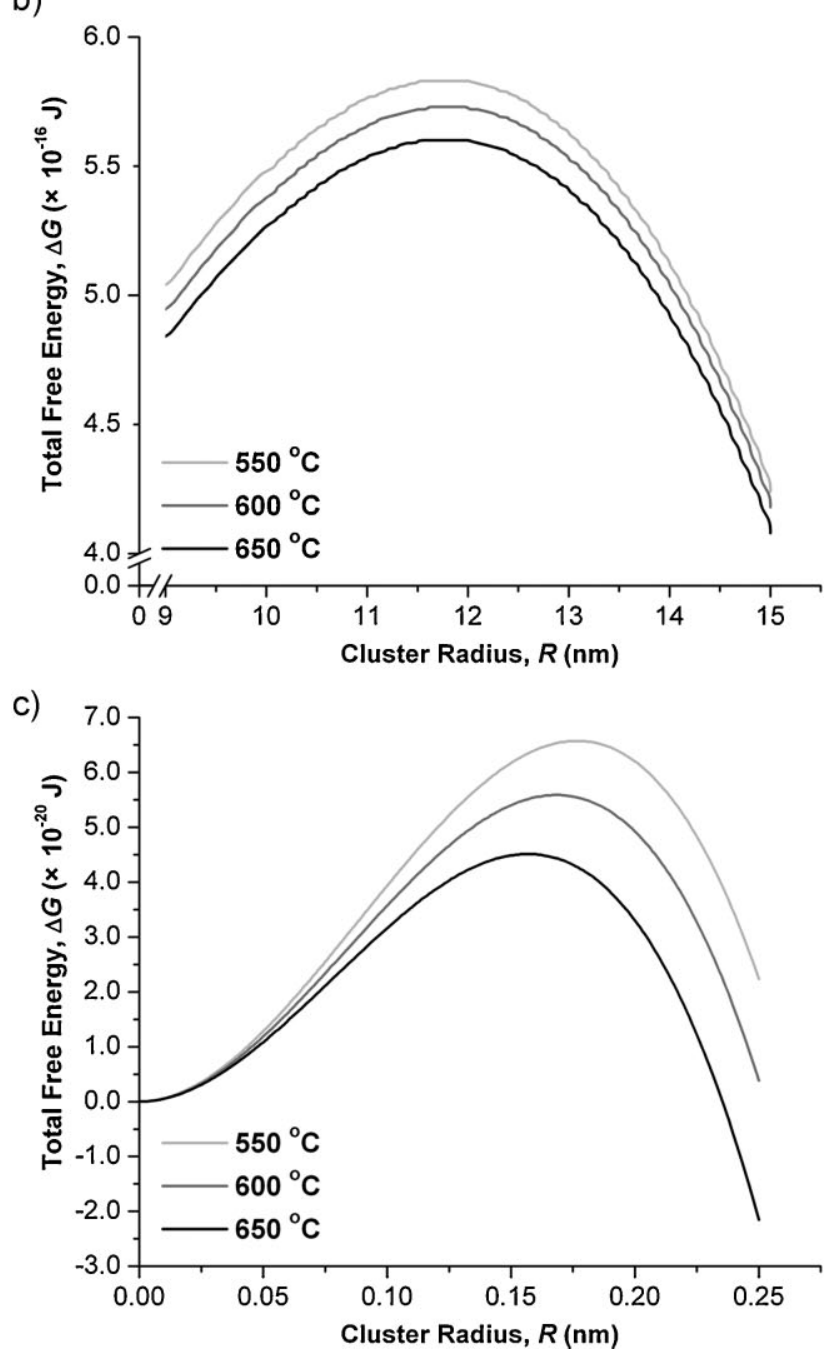

Figure 6. Analytical model of heterogeneous nucleation and growth of $\mathrm{ZnO}$ nanoclusters: a) diagram of a hemispherical $\mathrm{Zn}$ (or $\mathrm{ZnO}$ ) cluster being formed on a substrate surface, having a diameter of $R$ and consisting of $N \mathrm{Zn}$ atoms; plots (b) and (c) are showing $\Delta \mathrm{G}$ versus $R$ at different temperatures for the two limiting cases discussed in the text. Plot (b) applies when $\mathrm{Zn}$ vapor directly adsorbs to form $\mathrm{Zn}$ clusters which are later oxidized, and (c) applies when $\mathrm{Zn}$ vapor forms $\mathrm{ZnO}$ clusters immediately.
(CNT) curvature and/or the nanoscale geometry of the nucleation sites, and the geometry of the $\mathrm{ZnO}-\mathrm{CNT}$ interface will certainly determine the contact area per unit volume, and hence alter the contribution the $\mathrm{ZnO}-\mathrm{CNT}$ interface to the total free energy.

With these limitations in mind, we estimate the critical radius $R^{*}$ for two limiting cases: (I) where $\mathrm{Zn}$ vapor adsorbs to form $\mathrm{Zn}$ clusters which are later oxidized, and therefore $P_{\infty}$ is the vapor pressure of $\mathrm{Zn}$ at the substrate temperature; and (II) where $\mathrm{Zn}$ vapor forms $\mathrm{ZnO}$ clusters immediately, and therefore $P_{\infty}$ is the vapor pressure of $\mathrm{Zn}$ above $\mathrm{ZnO}$ at the substrate temperature. Substituting these and other parameter values ${ }^{[52]}$ for our growth conditions as described in the Supporting Information, we get $R^{*}$ $\approx 11.8 \mathrm{~nm}$ for case (I) and $\approx 0.17 \mathrm{~nm}$ for case (II). Also, as $T$ increases and temperature-dependent parameters such as surface energy and vapor pressure therefore also change accordingly, $\Delta \mathrm{G}$ decreases for both cases (Fig. 6b and 6c).

According to these calculations, the critical cluster size is substantially smaller than the ZNW diameter observed in TEM images. Therefore, we can make two conclusions: (1) the cluster size does not directly determine the ZNW diameter, and (2) VS growth of $\mathrm{ZnO}$ on CNTs reflects competition between NW growth
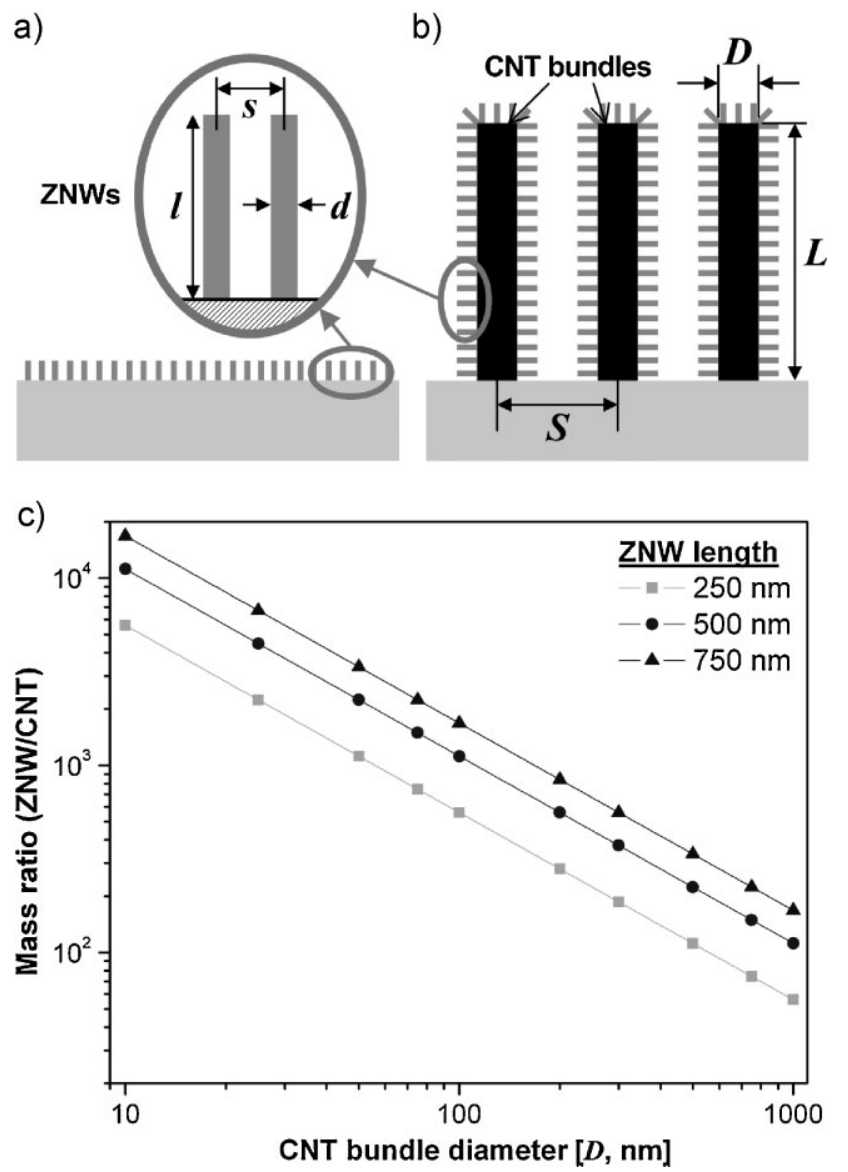

Figure 7. Increase in the yield of ZNWs and mass ratio (ZNW to CNT) achievable by introducing the ZNW/CNT hybrid architecture: comparative illustrations of the yield of ZNWs in (a) a flat substrate and (b) a CNT array. c) Calculated mass ratio of ZNWs to CNTs in the hybrid architecture. Parameters shown in (a) and (b) are defined in Figure S7 in the Supporting Information, and are used to plot (c). 
a)

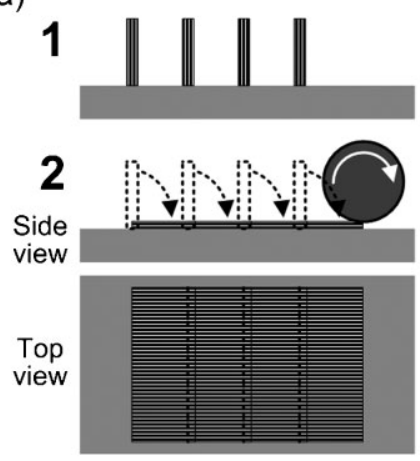

3

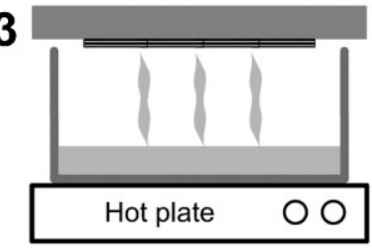

4

5

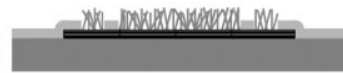

b)
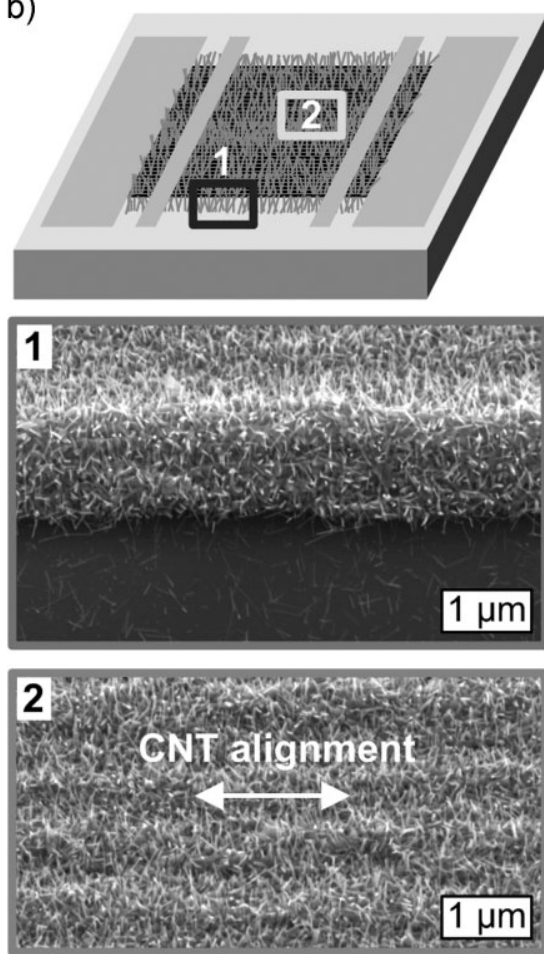

Figure 8. Fabrication and characterization of a hybrid ZNW/CNT film for measurement of electrical and photoelectric properties. Schematic (a) depicts (1) CNT forest blade growth from lithographically patterned catalyst; (2) and (3) folding of CNTs to horizontal orientation using mechanical rolling and surface tension; (4) deposition of Au contacts by using shadow mask pattern; and (5) ZNW growth. Schematic of the final device (b) is accompanied by SEM images of ZNW morphology on CNT sheet with $\approx 1.5 \mu \mathrm{m}$ thickness.

and polycrystalline film growth. As time proceeds, the balance between NW and film growth depends on the tendency to form new crystals via continuous nucleation, and the tendency to grow NWs from the initial crystals as their size increases, and clusters of a certain size much larger than the critical size for nucleation emerge in the [0001] direction as NWs.

Further, we observed that film growth dominates over NW growth as the temperature increases (Fig. S6). This corroborates the analytical prediction that the free energy of cluster formation $\Delta \mathrm{G}$ decreases as Tincreases; an increased tendency to form stable clusters would result in decreased mobility of $\mathrm{Zn}$ and/or $\mathrm{ZnO}$ atoms to the tips of NWs. In the future, measurement of the growth kinetics and diameter and length distributions of ZNWs under different process conditions will shed more light onto the factors controlling the nucleation density and size of the nanostructures on CNTs.

\section{Fabrication and Electrical Characterization of Anisotropic ZNW/CNT Thin Films}

Owing to the high surface area of the CNTs, the hybrid ZNW/CNT configuration increases the yield of ZNWs by a factor of $10^{1}-10^{3}$ as compared to growth on a flat substrate of the same area. Also, the large mass ratio of ZNWs to CNTs, which will be imperative to achieve high performance for energy conversion applications, can in principle be tuned by over a broad range $\left(\sim 10^{2}-10^{4}\right)$ by controlling the CNT and ZNW dimensions within reasonable bounds. All these calculations are based on measurements of ZNW dimensions from TEM images and are detailed in the Supporting Information with Figure S7 and Table S2, and summarized in Figure 7. Therefore, this material may present a significant performance advantage if intimate electrical contact is established between the CNTs and ZNWs at a high density and over a large area.

To investigate the electrical characteristics of the ZNW/CNT hybrid architecture, and to demonstrate its energy harvesting properties, we fabricated thin film devices comprising laterally aligned films of the hybrid material with integrated electrical contacts. Figure 8a depicts the fabrication procedure which is described in detail in the Experimental section. Briefly, CNT microstructures were grown from lithographically patterned catalyst, then the CNTs were "rolled" to form a horizontally aligned CNT (HA-CNT) sheet, ${ }^{[53]}$ then Au electrodes were deposited using shadow masks, and finally ZNWs were grown on the CNTs. As seen in SEM images of the device (Fig. 8b), ZNWs selectively grow on the CNTs in comparison to the surrounding substrate (i.e., smooth $\mathrm{SiO}_{2} / \mathrm{Si}$ surface), confirming that (consistent with the VS mechanism) the substrate surface roughness plays an important role in heterogeneous nucleation. ${ }^{[47]}$ This attribute is further advantageous in self-patterning ZNW growth based on the substrate topography.

The electrical characteristics of the laterally aligned films were measured using a 4-point method before and after ZNW growth, both parallel and perpendicular to the CNT alignment direction. The $I-V$ curves are shown in Figure 9a. As expected from the anisotropic organization of the film, the electrical conductivity is significantly larger along the CNT axis ("parallel" device), than perpendicular to the CNT axis ("perpendicular" device). This unique anisotropic behavior represents transport where the resistance is dominated by the characteristics (e.g., wall structure and defects) along individual CNTs in the parallel configuration; and primarily by electron hopping ${ }^{[54]}$ between CNTsidewalls in the perpendicular configuration.

The electrodes are deposited prior to ZNW growth and therefore contact the CNTs "under" the ZNWs. Because the anisotropic electrical conductivity of the film persists after ZNW growth, we conclude that the ZNW growth process does not alter the properties of CNTs. This corroborates our TEM images (Fig. 2) that show intimate contact between the graphitic CNT walls and $\mathrm{ZnO}$ nanocrystals. Further, the electrical conductivity in both directions is slightly higher after ZNW growth, demonstrating that the $\mathrm{ZnO}$ nanostructures bridge the high-resistance contacts between neighboring CNTs, and can also possibly mitigate resistance at serious defects (i.e., kinks, breakages) along individual CNTs. Accordingly, the relative decrease in resistivity after ZNW growth 


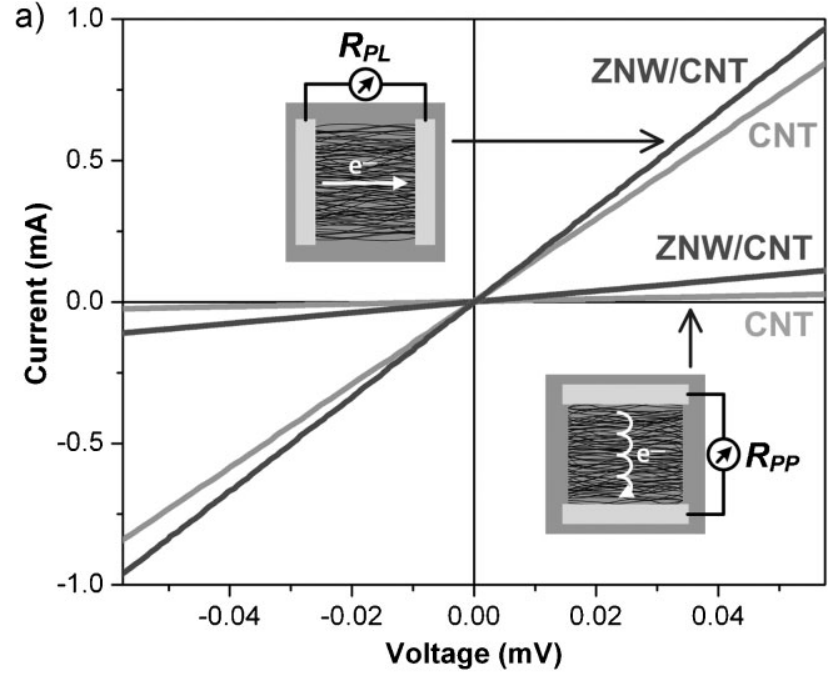

b)

\begin{tabular}{lc}
\multicolumn{2}{l}{ (I) Parallel } \\
\hline & $\begin{array}{c}\text { Resistivity } \\
{[\Omega-\mathrm{cm}]}\end{array}$ \\
\hline CNT & $1.65 \times 10^{-2}$ \\
\hline ZNW/CNT & $1.46 \times 10^{-2}$ \\
\hline
\end{tabular}

(II) Perpendicular

\begin{tabular}{lc}
\hline & $\begin{array}{c}\text { Resistivity } \\
{[\Omega-\mathrm{cm}]}\end{array}$ \\
\hline CNT & $6.02 \times 10^{-1}$ \\
\hline ZNW/CNT & $1.37 \times 10^{-1}$ \\
\hline
\end{tabular}

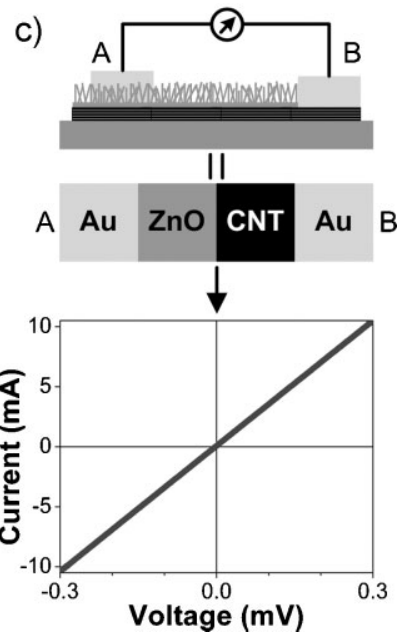

Figure 9. Anisotropic electrical characteristics of ZNW/CNT sheets: a) I-V curves before and after ZNW growth in the parallel (along the CNTs) and perpendicular (normal to the CNTs) configurations; b) resistivity data, based on I-V measurements and sheet geometry, before and after ZNW growth; and c) $I-V$ measurement after an additional Au electrode is deposited on top of the ZNW layer, as shown in the accompanying schematic. Insets to (a) schematically show electron transport mechanism through aligned CNTs in parallel and perpendicular directions.

(Fig. 9b) is much larger (77\%) for the perpendicular device than the parallel device $(11 \%)$. The resistivity of ZNWs was previously measured to be $3.5 \times 10^{6} \Omega \mathrm{cm},{ }^{[55]}$ which is several orders of magnitude higher than measured for the CNT sheet in either direction. Therefore, even though the electrodes are connected by a continuous path of ZnO because the CNTs are coated with a thin $\mathrm{ZnO}$ film at the base of the NWs, electrical transport through the $\mathrm{ZnO}$ has a negligible contribution to the transport through the device.

We also measured the electrical transport across the $\mathrm{ZnO} / \mathrm{CNT}$ interface by depositing a fifth Au electrode on top of the device after $\mathrm{ZNW}$ growth, resulting in transport through a series path of $\mathrm{Au}-$ $\mathrm{ZnO}-\mathrm{CNT}-\mathrm{Au}$. The linearity of this $I-V$ curve (Fig. 9c), in conjunction with the linearity of the $I-V$ curves through the CNTs (Au-CNT-Au; Fig. 9a) demonstrates Ohmic contact between $\mathrm{ZnO}$ and the CNTs. In other words, the $\mathrm{ZnO}$ and CNTs form a nonrectifying junction, which is expected ${ }^{[56]}$ because MWNTs, which are metallic regardless of their chirality, have a lower work function $\left(4.5-5.0 \mathrm{eV}^{[57]}\right)$ than n-type $\mathrm{ZnO}\left(5.3-5.4 \mathrm{eV}^{[58]}\right)$.

\section{Photoconductive Behavior and Photocurrent Generation of the ZNW/CNT Hybrids}

Finally, we demonstrate that the ZNW/CNT hybrids can be building blocks for new energy conversion materials that couple the attractive properties of ZNWs and CNTs. As an initial step in this direction, the hybrid ZNW/CNT films demonstrate photoconductive behavior and photocurrent generation, with rapid dynamics, upon exposure to UV illumination. These tests were performed using the ZNW/CNT film with $\mathrm{Au}$ contacts in the parallel device configuration, and the intensity of illumination was controlled by changing the distance between the UV source and the device. CNT sheets without ZNWs do no exhibit any of these photoresponsive characteristics. The configuration for these measurements is shown schematically in Figure 10a.

\subsection{Photoconductive Behavior}

First, in Figure 10b, we see that the resistivity of the ZNW/CNT film decreases slightly upon UV illumination, and the magnitude of this decrease is proportional to the UV intensity. This is wellknown as the photoconductive effect, ${ }^{[5]}$ and the relatively small magnitude of the decrease in resistivity is consistent with our understanding that the electrical transport through the device is dominated by conduction through the underlying MWNTs which are metallic. Based on previous studies of $\mathrm{ZnO}$ photoconduction, ${ }^{[59-61]}$ we suggest that ambient $\mathrm{O}_{2}$ adsorbs on the $\mathrm{ZNW}$ surfaces and associates with free electrons from the n-type $\mathrm{ZnO}$. When the device is illuminated with a photon energy exceeding the $\mathrm{ZnO}$ band gap $(\approx 3.37 \mathrm{eV})$, electron-hole pairs are generated in $\mathrm{ZnO}$. The photogenerated holes migrate to the surface along the potential slope created by band bending and discharge the adsorbed $\mathrm{O}_{2}$ ion.

$h^{+}+\mathrm{O}_{2}^{-}(a d) \rightarrow \mathrm{O}_{2}(\mathrm{~g})$

The remaining free electrons enhance the conductivity of $\mathrm{ZnO}$, and this marginally enhances the ZNW/CNT hybrid conductivity.

Previous photoconductivity measurements of individual ZNWs made electrical contacts directly to the $\mathrm{ZnO}$, which in combination with the low conductivity of $\mathrm{ZnO}$ results in significant photoconductive gains. ${ }^{[60]}$ However, the comparatively slight enhancement of the ZNW/CNT conductivity in our study suggests that the additional free electrons are donated from the $\mathrm{ZnO}$ nanostructures to the CNTs, and these electrons have a measurable yet small effect on the CNT conductivity. Consistent with the photoconduction mechanism, there is no measurable change in conductivity when the ZNW/CNT device is exposed to UV light in vacuum (Fig. S8). Therefore, in the absence of oxygen interaction on the surface of $\mathrm{ZnO}$, the photogenerated electronhole pairs recombine within the material and do not contribute to conduction. 
a)

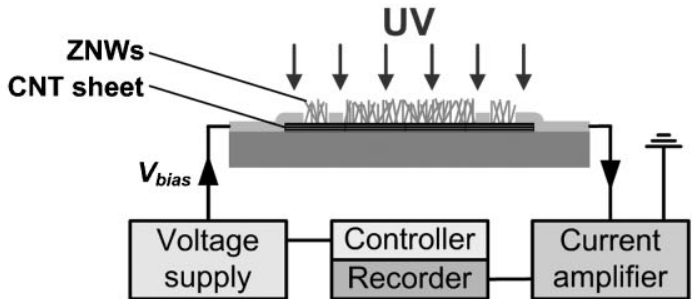

b)

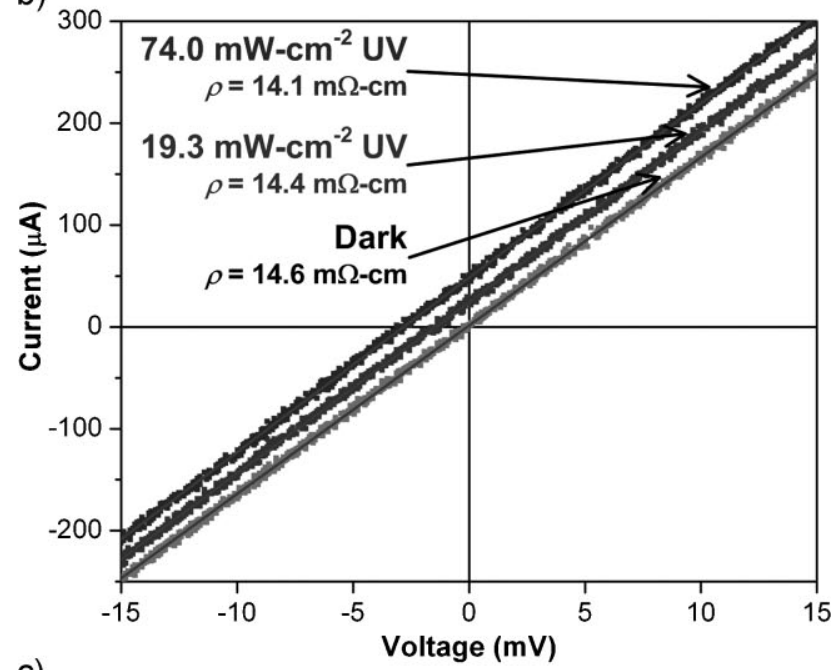

c)

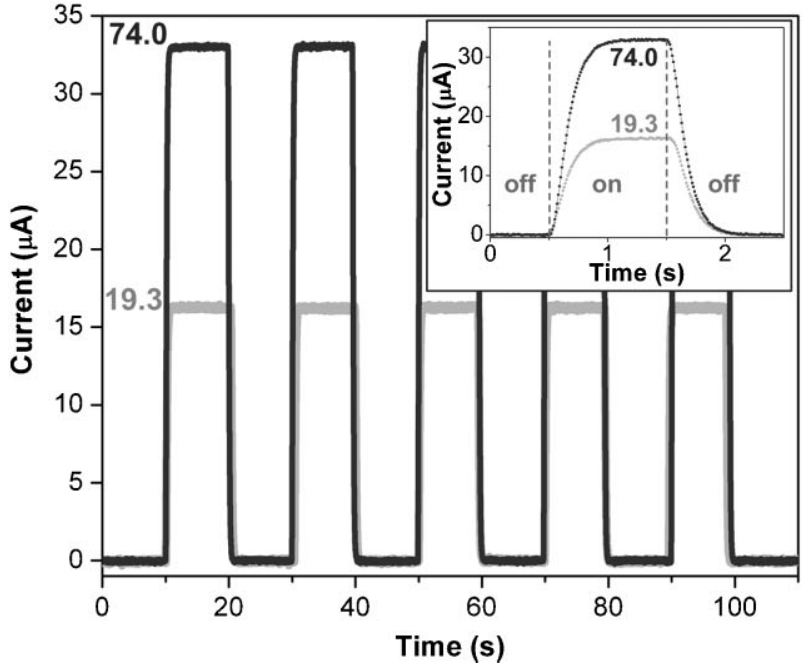

Figure 10. Photoelectric behavior of a hybrid ZNW/CNT thin-film device in the parallel configuration: a) measurement configuration; b) $I-V$ curves upon $365 \mathrm{~nm}$ UV irradiation at different intensities; c) time-resolved photocurrent generated by turning on and off the UV light without applied bias. Inset to (c) shows a single curve during $1 \mathrm{~s}$ UV exposure, demonstrating rapid photoresponse $(\approx 0.5 \mathrm{~s})$.

\subsection{Photocurrent Generation and Response Dynamics}

The ZNW/CNT film also produces a distinct electrical current when illuminated under zero bias, indicating that the hybrid material itself operates like a photovoltaic cell (Fig. 10c). We suggest this current is generated by continuous turnover of the oxygen-mediated photoconduction mechanism. Continuous adsorption and desorption of ionized surface oxygen collects photogenerated holes from the surface of the $\mathrm{ZnO}$ (the anode), while electrons flow into the CNTs (the cathode) and are collected by the circuit. Notably, this current is absent in vacuum, confirming that ambient oxygen is necessary to generate the photocurrent. The high surface area of our device increases the intensity of the photocurrent, even though the kinetics of oxygen adsorption and desorption may be relatively slow. The external quantum efficiency (EQE) of this behavior is approximately $1 \%$, at a UV intensity of $19.3 \mathrm{~mW} \mathrm{~cm}^{-2}$. Under these conditions, the device generates $5.93 \mu \mathrm{A} \mathrm{cm}^{-2}$ (based on the illuminated film area of $0.27 \mathrm{~cm}^{2}$ ), and $0.97 \mathrm{mV}$ which is measured across the $\mathrm{Au}$ electrodes at opposite extents of the film.

The ability of the hybrid material to generate a photocurrent through the CNTs at zero bias indicates that electrons from the semiconducting $\mathrm{ZnO}$ structures can readily transport to the metallic CNTs due to the ohmic contact between the CNTs and $\mathrm{ZnO}$. Upon exciton generation inside the $\mathrm{ZnO}$, photogenerated electrons from $\mathrm{ZnO}$ flow into the CNTs. The direction of the photocurrent is determined by the contact resistances between the $\mathrm{Au}$ electrodes and the ZNW/CNT film. The photocurrent flows to the electrode with the lowest contact resistance, and the direction of current flow does not change when the connections to the measurement circuit are reversed.

Finally, the photoresponse dynamics are studied by switching the UV light on and off, with the device in air. The rates of increase and decay of the photocurrent are strikingly rapid, as it takes less than $0.5 \mathrm{~s}$ for the current to decay to below $1 \%$ of the steady illumination value at $0 \mathrm{~V}$ bias (inset to Fig. 10c), and less than $1 \mathrm{~min}$ to decay at $300 \mathrm{mV}$ bias (data not shown). Comparatively, UV photodetectors based on individual ZNWs exhibit response times ranging from seconds to minutes, ${ }^{[5,60,62]}$ and photodetectors using polycrystalline $\mathrm{ZnO}$ thin films exhibit response times ranging from a minutes to hours. ${ }^{[61]}$ The rapid response of the ZNW/CNT hybrid is attributed to the intimate contact between the ZNWs and CNTs, which enables rapid dissipation of carriers that remain in the $\mathrm{ZnO}$ after the illumination is turned off. Hence, the distance for dissipation of charge carriers is the ZNW length, and therefore our material combines the large-area scalability of a thin film with the rapid response observed with individual NWs.

\section{Conclusions}

We have demonstrated a suite of versatile methods for building hybrid architectures of ZNWs on aligned CNTs. The inherent scalability of the two-step CVD process, as well as the rolling and patterning methods used to fabricate functional hybrid sheets, suggest it will be possible to scale this process for efficient manufacturing. The CNTs are a true 3D substrate that makes uniform and distributed electrical contact to the ZNWs, thereby overcoming an important obstacle to the use of aligned NWs that have been previously demonstrated on flat substrates. The UVinduced photoconductive behavior and photocurrent generation of the ZNW/CNT hybrids are examples of how this architecture may be used to efficiently couple the attractive properties of NWs in large numbers. These may take advantage of the utility of ZNWs in dye-sensitized solar cells, gas sensors, and piezoelectric devices. 
Our process is also scalable to virtually any CNT configuration, ranging from isolated CNTs in electronic devices, to bulk configurations like tangled films ("buckypapers") and powders. These are important steps toward next-generation nanoscale energy conversion and storage technologies.

\section{Experimental}

CNT Forest Growth [63]: Catalyst and support layers of Fe $(1 \mathrm{~nm})$ and $\mathrm{Al}_{2} \mathrm{O}_{3}(10 \mathrm{~nm})$ were sequentially deposited by electron beam evaporation (S)-26, Denton Vacuum, Inc.) onto a p-type (100) silicon wafer coated with $500-n m$-thick thermally grown $\mathrm{SiO}_{2}$. The catalyst substrate was loaded in a 1 in. diameter quartz tube and placed in a single-zone atmospheric pressure tube furnace (Thermo-Fisher MiniMite), which is shown schematically in Figure S2a. The sample was located $10 \mathrm{~cm}$ downstream of the control thermocouple of the furnace. After purging with $1000 \mathrm{sccm} \mathrm{He}(99.999 \%$ UHP, Metro Welding, Detroit, MI) at room temperature for $5 \mathrm{~min}$, a flow of $400 \mathrm{sccm} \mathrm{He}$ and $100 \mathrm{sccm} \mathrm{H}$ (99.999\% UHP, Metro Welding) was maintained for $5 \mathrm{~min}$ to establish the catalyst annealing atmosphere before heating. Then, the furnace was heated to the growth temperature of $775^{\circ} \mathrm{C}$ for $10 \mathrm{~min}$, and maintained for $10 \mathrm{~min}$ to anneal the catalyst, and then $100 \mathrm{sccm} \mathrm{C}_{2} \mathrm{H}_{4}$ (99.9\% UHP, Airgas, Inc., Randor, PA) was added for the growth duration of $20 \mathrm{~min}$. After growth, the furnace was cooled to room temperature under a slight $\mathrm{He}$ flow.

ZnO NW Growth on CNTs: ZnO NW (ZNW) growth was performed in the same tube furnace as CNT growth, and separate dedicated quartz tubes were used for CNT and ZNW growth. A piece of Zn foil $(\approx 0.6 \mathrm{~g}, 99.98 \%$, Alfa Aesar) was loaded at the center position (location of control thermocouple) the of the 1 in. diameter quartz tube and the CNT samples (i.e., plain CNT forests, CNT bundles on TEM grids, horizontally aligned CNT sheets) were placed $6-7 \mathrm{~cm}$ downstream of the center. The furnace was initially pumped to approximately $10^{-2}$ Torr, then was heated at a ramp rate of $30^{\circ} \mathrm{C} \mathrm{min}-1$ under a controlled amount of He flow, which was kept throughout the process to facilitate vapor transport. Then, a flow of air (zero grade, Airgas) was added after the furnace temperature surpassed the melting temperature of $\mathrm{Zn}\left(\approx 420^{\circ} \mathrm{C}\right)$; this initiates $\mathrm{ZNW}$ formation onto the target substrates [37]. The pressure was maintained constant throughout the process using a feedback control system with a motorized valve.

The structures shown in Figures 1-6 were grown at $17.5 / 20 \mathrm{sccm}$ air/ $\mathrm{He}$, at $6 \mathrm{Torr}$ pressure, with $600^{\circ} \mathrm{C}$ furnace setpoint temperature. According to temperature profiles (Fig. S2b) measured along the central axis of the furnace tube, the temperature was $614^{\circ} \mathrm{C}$ at the center location and $591{ }^{\circ} \mathrm{C}$ at the sample position.

Structural and Optical Characterization: SEM imaging along with EDX analysis was performed using a Philips XL30-FEG, operating at $10 \mathrm{kV}$, with the stage typically tilted at $45^{\circ}$. GI-XRD analysis was conducted under thin film grazing incidence mode at $\mathrm{Gl}$ angle of $0.5^{\circ}$ with $\mathrm{Cu} \mathrm{K}$ (alpha) laser radiation (Rigaku Ultima IV). To prepare samples for TEM characterization, as-grown CNTs were sonicated in toluene without any surfactant for $1 \mathrm{~h}$ and several drops of this dispersion were applied to a copper mesh TEM grid. After drying in flowing $\mathrm{N}_{2}$, some of the thin CNT bundles and/or individual CNT filaments remained suspended. ZNWs were then grown on the CNTs by placing the grid inside the furnace at the same position as the flat substrates. A JEOL 3011 TEM instrument operating at $300 \mathrm{keV}$ was used for conventional TEM (CTEM) imaging. A JEOL-2010F analytical electron microscope (AEM) supporting both CTEM and STEM modes was used at the operation voltage of $200 \mathrm{keV}$ to characterize the $\mathrm{ZnO}-\mathrm{CNT}$ interface and its elemental composition through high-angle annular darkfield (HAADF) imaging and EELS. The lens conditions were set to define a probe size of $0.2 \mathrm{~nm}$ for HAADF imaging and EELS analysis. A $0.5 \mathrm{eV} /$ pixel dispersion was used that gave an energy resolution of about $1.5 \mathrm{eV}$ (fullwidth at half maximum, FWHM). X-ray diffraction was performed on a Rigaku Ultima IV in thin film grazing incidence (GI) mode at an angle of $0.5^{\circ}$.
In preparation for $\mathrm{PL}$ measurements, ZNWs were first grown on a $1 \times 1 \mathrm{~cm}$ CNT forest substrate, and then the ZNW/CNT sample was carefully delaminated from the substrate using a cleaned razor blade. The ZNW/CNT forest was mounted on a quartz plate using a double-sided tape $(3 \mathrm{M})$. An as-grown CNT sample with the same size was processed identically as a reference. The PL spectra were acquired using a fluorescence spectrophotometer (QM-4, PTI) with an excitation beam wavelength of $325 \mathrm{~nm}$.

Device Fabrication: First, vertically aligned CNT "blades" were grown from lithographically patterned catalyst. To pattern the catalyst, a $\mathrm{SiO}_{2} / \mathrm{Si}$ wafer was coated with $\approx 3-\mu \mathrm{m}$-thick photoresist (SPR220) by spin-coating at $3000 \mathrm{rpm}$ for $30 \mathrm{~s}$ and pre-baking at $115^{\circ} \mathrm{C}$ for $90 \mathrm{~s}$. The sample was exposed to UV light for $6 \mathrm{~s}$ on a Karl Suss MA-6 Mask Aligner in order to lithographically define the shape and position of the catalysts. The sample was then post-baked at $115^{\circ} \mathrm{C}$ for $90 \mathrm{~s}$, and then immersed in MIF 300 developer for $1 \mathrm{~min}$. Next, $\mathrm{Fe}$ and $\mathrm{Al}_{2} \mathrm{O}_{3}$ were deposited by e-beam evaporation on the sample. Lift-off was performed by sonicating the sample in acetone for 5-10 min, then washing in isopropyl alcohol and deionized water. After CNT growth, the CNT blades were transformed to horizontally aligned (HA-CNT) sheets by mechanical rolling followed by elastocapillary densification as discussed in our separate publication [53]. Electrodes were then deposited on by e-beam evaporation of $\mathrm{Au}(800 \mathrm{~nm}$ thickness) through shadow masks that were laser-cut in polyester film. Finally, ZNWs were grown directly on the CNT sheet device having Au electrodes by the aforementioned procedure.

Device Characterization: The electrical and photoelectric properties of ZNW/CNT devices were characterized via a 4-point $I-V$ measurement using a custom-built probe station. UV irradiation was applied using a UV lamp (Dymax, Model 2000 Flood, $365 \mathrm{~nm}, 75 \mathrm{~mW}-\mathrm{cm}^{-2}$ ). The UV intensity was modulated by changing the distance between the source and the device. For the measurements in vacuum environment, a vacuum probe station equipped with an ultrahigh vacuum system (Varian, TPS-Compact) and an optical window was used, and the experiments were carried out at the pressure lower than $2.5 \times 10^{-6}$ Torr. All UV exposure experiments were conducted in a dark room to exclude any external light disturbance to the measurements.

\section{Acknowledgements}

This work was funded by the University of Michigan, Department of Mechanical Engineering and College of Engineering, and the DARPA Microsystems Technology Office through a Young Faculty Award to A.J.H.. J.G.O. and S.H.T. were supported in part by University of Michigan Mechanical Engineering Departmental Fellowships. We thank John Mansfield for assistance with HR-SEM imaging, Jiseok Lee, Onas Bolton, and Prof. Jinsang Kim for assistance with PL measurements, Sudhanshu Srivastava and Prof. Nicholas A. Kotov for instructive discussions on PL characterization, Sei-jin Park for shadow mask preparation, and Chung Chiang Wu, Girish Kulkarni, Nanditha Dissanayake, and Prof. Zhaohui Zhong for use of their electrical testing facilities and assistance with electrical characterization. Electron microscopy was performed at the University of Michigan Electron Microbeam Analysis Laboratory (EMAL), and microfabrication was performed at the University of Michigan Lurie Nanofabrication Facility (LNF). Supporting Information is available online from Wiley InterScience or from the author.

Received: February 5, 2010 Revised: March 26, 2010 Published online: June 24, 2010

[1] D. R. Rolison, R. W. Long, J. C. Lytle, A. E. Fischer, C. P. Rhodes, T. M. McEvoy, M. E. Bourga, A. M. Lubers, Chem. Soc. Rev. 2009, 38, 226.

[2] Y. N. Xia, P. D. Yang, Y. G. Sun, Y. Y. Wu, B. Mayers, B. Gates, Y. D. Yin, F. Kim, Y. Q. Yan, Adv. Mater. 2003, 15, 353.

[3] W. Lu, C. M. Lieber, J. Phys. D: Appl. Phys. 2006, 39, R387.

[4] L. D. Qin, S. Park, L. Huang, C. A. Mirkin, Science 2005, 309, 113. 
[5] F. S. Ou, M. M. Shaijumon, L. Ci, D. Benicewicz, R. Vajtai, P. M. Ajayan, Appl. Phys. Lett. 2006, 89, 243122.

[6] a) B. Z. Tian, X. L. Zheng, T. J. Kempa, Y. Fang, N. F. Yu, G. H. Yu, J. L. Huang, C. M. Lieber, Nature 2007, 449, 885. b) B. Tian, T. J. Kempa, C. M. Lieber, Chem. Soc. Rev. 2009, 38, 16.

[7] a) M. J. Bierman, Y. K. A. Lau, S. Jin, Nano Lett. 2007, 7, 2907. b) K. A. Dick, K. Deppert, M. W. Larsson, T. Martensson, W. Seifert, L. R. Wallenberg, L. Samuelson, Nat. Mater. 2004, 3, 380; K. A. Dick, S. Kodambaka, M. C. Reuter, K. Deppert, L. Samuelson, W. Seifert, L. R. Wallenberg, F. M. Ross, Nano Lett. 2007, 7, 1817.

[8] M. J. Bierman, Y. K. A. Lau, A. V. Kvit, A. L. Schmitt, S. Jin, Science 2008, 320, 1060.

[9] a) J. H. Zou, S. I. Khondaker, Q. Huo, L. Zhai, Adv. Funct. Mater. 2009, 19, 479. b) A. L. M. Reddy, M. M. Shaijumon, S. R. Gowda, P. M. Ajayan, Nano Lett. 2009, 9, 1002. c) A. J. Miller, R. A. Hatton, S. R. P. Silva, Appl. Phys. Lett. 2006, 89, 023 105. d) B. Li, L. Y. Li, B. B. Wang, C. Y. Li, Nat. Nanotechnol. 2009, 4, 358

[10] C. Masarapu, V. Subramanian, H. W. Zhu, B. Q. Wei, Adv. Funct. Mater 2009, 19, 1008.

[11] V. L. Pushparaj, M. M. Shaijumon, A. Kumar, S. Murugesan, L. Ci, R. Vajtai, R. J. Linhardt, O. Nalamasu, P. M. Ajayan, Proc. Natl. Acad. Sci. USA 2007, 104, 13574.

[12] D. Tasis, N. Tagmatarchis, A. Bianco, M. Prato, Chem. Rev. 2006, 106, 1105.

[13] a) Carbon Nanotubes: Synthesis, Structure, Properties, and Applications (Eds: M. S. Dresselhaus, G. Dresselhaus, P. Avouris ) Springer, New York 2001. b) M. Endo, T. Hayashi, Y. A. Kim, M. Terrones, M. S. Dresselhaus, Philos. Trans. R. Soc. A 2004, 362, 2223.

[14] S. S. Fan, M. G. Chapline, N. R. Franklin, T. W. Tombler, A. M. Cassell, H. J. Dai, Science 1999, 283, 512 .

[15] T. Hiraoka, T. Yamada, K. Hata, D. N. Futaba, H. Kurachi, S. Uemura, M. Yumura, S. lijima, J. Am. Chem. Soc. 2006, 128, 13338.

[16] N. Yamamoto, A. J. Hart, E. J. Garcia, S. S. Wicks, H. M. Duong, A. H. Slocum, B. L. Wardle, Carbon 2009, 47, 551; L. T. Qu, Y. Zhao, L. M. Dai, Small 2006, 2, 1052.

[17] A. J. Hart, A. H. Slocum, Nano Lett. 2006, 6, 1254

[18] a) R. Z. Ma, J. Liang, B. Q. Wei, B. Zhang, C. L. Xu, D. H. Wu, J. Power Sources 1999, 84, 126. b) D. N. Futaba, K. Hata, T. Yamada, T. Hiraoka, Y. Hayamizu, Y. Kakudate, O. Tanaike, H. Hatori, M. Yumura, S. lijima, Nat. Mater. 2006, 5, 987.

[19] H. Ago, K. Petritsch, M. S. P. Shaffer, A. H. Windle, R. H. Friend, Adv. Mater. 1999, 11, 1281.

[20] M. H. Huang, Y. Y. Wu, H. Feick, N. Tran, E. Weber, P. D. Yang, Adv. Mater. 2001, 13, 113.

[21] M. H. Huang, S. Mao, H. Feick, H. Q. Yan, Y. Y. Wu, H. Kind, E. Weber, R. Russo, P. D. Yang, Science 2001, 292, 1897.

[22] Z. L. Wang, J. H. Song, Science 2006, 312, 242.

[23] S. T. Ho, K. C. Chen, H. A. Chen, H. Y. Lin, C. Y. Cheng, H. N. Lin, Chem. Mater. 2007, 19, 4083.

[24] B. Weintraub, Y. L. Deng, Z. L. Wang, J. Phys Chem. C 2007, 111, 10162.

[25] S. Xu, Y. G. Wei, J. Liu, R. Yang, Z. L. Wang, Nano Lett. 2008, 8, 4027.

[26] J. T. Hu, M. Ouyang, P. D. Yang, C. M. Lieber, Nature 1999, 399, 48.

[27] J. Luo, L. Zhang, Y. J. Zhang, J. Zhu, Adv. Mater. 2002, 14, 1413.

[28] J. Luo, Z. P. Huang, Y. G. Zhao, L. Zhang, J. Zhu, Adv. Mater. 2004, 16, 1512.

[29] R. Y. Li, X. C. Sun, X. R. Zhou, M. Cai, X. L. Sun, J. Phys Chem C 2007, 111, 9130.

[30] V. Subramanian, H. W. Zhu, B. Q. Wei, Electrochem. Commun. 2006, 8, 827.

[31] H. L. Hsiao, Z. Y. Liu, P. H. Lee, at. presented, 2nd International Conference on New Diamond and Nano Carbons, Taipei, Taiwan, May 2008.

[32] S. Sun, D. Yang, G. Zhang, E. Sacher, J. P. Dodelet, Chem. Mater. 2007, 19 , 6376.
[33] J. W. Liu, Y. T. Kuo, K. J. Klabunde, C. Rochford, J. Wu, J. Li, ACS Appl. Mater. Q Interfaces 2009, 1, 1645.

[34] S. H. Dalal, D. L. Baptista, K. B. K. Teo, R. G. Lacerda, D. A. Jefferson, W. I. Milne, Nanotechnology 2006, 17, 4811.

[35] W. D. Zhang, Nanotechnology 2006, 17, 1036.

[36] a) H. Liu, S. H. Li, J. Zhai, H. J. Li, Q. S. Zheng, L. Jiang, D. B. Zhu, Angew. Chem. Int. Ed. 2004, 43, 1146. b) C. Py, R. Bastien, J. Bico, B. Roman, A. Boudaoud, Europhys. Lett. 2007, 77, 44005.

[37] K. Wang, J. J. Chen, W. L. Zhou, Y. Zhang, Y. F. Yan, J. Pern, A. Mascarenhas, Adv. Mater. 2008, 20, 3248

[38] a) P. D. Yang, H. Q. Yan, S. Mao, R. Russo, J. Johnson, R. Saykally, N. Morris, J. Pham, R. R. He, H. J. Choi, Adv. Funct. Mater. 2002, 12, 323 b) X. D. Wang, C. J. Summers, Z. L. Wang, Nano Lett. 2004, 4, 423.

[39] Z. Y. Jiang, Z. X. Xie, X. H. Zhang, S. C. Lin, T. Xu, S. Y. Xie, R. B. Huang, L. S. Zheng, Adv. Mater. 2004, 16, 904.

[40] Z. L. Wang, J. Phys.: Cond. Matter 2004, 16, R829.

[41] E. Einarsson, H. Shiozawa, C. Kramberger, M. H. Rummeli, A. Gruneis, T. Pichler, S. Maruyama, J. Phys. Chem. C 2007, 111, 17861; E. Verploegen, G. Chia, S. Tawfick, M. De Volder, A. J. Hart, R. E. Cohen, unpublished.

[42] a) R. D. Bennett, A. J. Hart, R. E. Cohen, Adv. Mater. 2006, 18, 2274 b) G. D. Nessim, A. J. Hart, J. S. Kim, D. Acquaviva, J. H. Oh, C. D. Morgan, M. Seita, J. S. Leib, C. V. Thompson, Nano Lett. 2008, 8, 3587.

[43] A. B. Djurisic, Y. H. Leung, Small 2006, 2, 944.

[44] K. Vanheusden, W. L. Warren, C. H. Seager, D. R. Tallant, J. A. Voigt, B. E. Gnade, J. Appl. Phys. 1996, 79, 7983.

[45] S. Chakrabarti, D. Ganguli, S. Chaudhuri, J. Phys. D: Appl. Phys. 2003, 36, 146.

[46] G. W. Sears, Acta Metall. 1955, 3, 361.

[47] S. T. Ho, C. Y. Wang, H. L. Liu, H. N. Lin, Chem. Phys. Lett. 2008, 463, 141

[48] a) W. K. Burton, N. Cabrera, F. C. Frank, Nature 1949, 163, 398. b) W. K. Burton, N. Cabrera, F. C. Frank, Phil. Trans. R. Soc. A 1951, 243, 299. c) J. D. Eshelby, J. Appl. Phys. 1953, 24, 176; G. W. Sears, Acta Metall. 1953, 1, 457.

[49] a) H. Heyer, Angew. Chem. Int. Ed. 1966, 5, 67. b) R. L. Parker R. L. Anderson, S. C. Hardy, Appl. Phys. Lett. 1963, 3, 93.

[50] J. B. Baxter, E. S. Aydil, J. Cryst. Growth 2005, 274, 407.

[51] R. W. Balluffi, S. M. Allen, W. C. Carter, Kinetics of Materials, John Wiley \& Sons, Inc., Hoboken, NJ 2005

[52] a) T. R. Hogness, J. Am. Chem. Soc. 1921, 43, 1621. b) C. G. Maier, J. Am. Chem. Soc. 1926, 48, 356. c) D. Segets, J. Gradl, R. K. Taylor, V. Vassilev, W. Peukert, ACS Nano 2009, 3, 1703 . d) R. H. Lamoreaux D. L. Hildenbrand, L. Brewer, J. Phys. Chem. Ref. Data 1987, 16, 419.

[53] S. Tawfick, K. O'Brien, A. J. Hart, Small 2009, 5, 2467.

[54] C. L. Pint, Y. Q. Xu, E. Morosan, R. H. Hauge, Appl. Phys. Lett. 2009, 94 182107.

[55] H. Kind, H. Q. Yan, B. Messer, M. Law, P. D. Yang, Adv. Mater. 2002, 14, 158.

[56] D. A. Neamen, Semiconductor Physics and Devices: Basic Principles, McGrawHill, New York 2003

[57] a) M. Shiraishi, M. Ata, Carbon 2001, 39, 1913; H. Ago, T. Kugler, F. Cacialli, W. R. Salaneck, M. S. P. Shaffer, A. H. Windle, R. H. Friend, J. Phys. Chem. B 1999, 103, 8116

[58] S. H. Jo, D. Banerjee, Z. F. Ren, Appl. Phys. Lett. 2004, 85, 1407.

[59] Y. Takahashi, M. Kanamori, A. Kondoh, H. Minoura, Y. Ohya, Jpn, J. Appl. Phys, Part 1: Regular Pap. Short Notes a Rev. Pap. 1994, 33, 6611.

[60] C. Soci, A. Zhang, B. Xiang, S. A. Dayeh, D. P. R. Aplin, J. Park, X. Y. Bao, Y. H. Lo, D. Wang, Nano Lett. 2007, 7, 1003.

[61] Y. Z. Jin, J. P. Wang, B. Q. Sun, J. C. Blakesley, N. C. Greenham, Nano Lett. 2008, 8, 1649.

[62] W. Kim, K. S. Chu, Phys. Status Solidi A 2009, 206, 179.

[63] A. J. Hart, A. H. Slocum, J. Phys. Chem. B 2006, 110, 8250. 